\title{
Integración de la computación física bajo el aprendizaje construccionista para el fortalecimiento de las competencias tecnológicas de los docentes del TecNM campus Minatitlán
}

\section{Integration of physical computing under constructionist learning to strengthen the technological competencies of the teachers of the TecNM campus Minatitlán}

\author{
Guillermina Jiménez Rasgado ${ }^{1}$, Claudia Marina Vicario Solórzano ${ }^{2}$ \\ guillejr10@gmail.com, marina.vicario@gmail.com \\ ${ }^{1}$ Ingeniería Electrónica \\ Tecnológico Nacional de México-Minatitlán \\ Minatitlán, Veracruz, México. \\ ${ }^{2}$ Nodo UPIICSA de la Red de Computación \\ Instituto Politécnico Nacional / Red LaTE Mx \\ Ciudad de México, México.
}

\begin{abstract}
Resumen. El presente trabajo de investigación fue antes de la pandemia COVID-19 tuvo como objetivo valorar las posibilidades de aplicación y de integración de la computación física, mediante la programación de sensores y actuadores para contribuir con el fortalecimiento de conocimientos, competencias y uso de nuevas herramientas tecnológicas para los docentes de la carrera de Ingeniería Electrónica del Tecnológico Nacional de México campus Minatitlán, aplicando la teoría construccionista y la herramienta tecnológica de Arduino Uno, se diseñaron prácticas, y se aplicó la prueba de conceptos mediante un taller presencial. Aplicando al final dos encuesta. En esta investigación, se concluye que la Computación física con Arduino Uno bajo el enfoque construccionista, fortalece diversas competencias y conocimientos relacionados a la programación, pero también se desarrollan habilidades tangibles como las de diseñar, conectar, experimentar y construir, así como se favorecen habilidades suaves tal como la colaboración, el pensamiento computacional, la creatividad, comunicación y la resolución de problemas.
\end{abstract}

\section{Palabras clave: Computación física, Construccionismo, Edmodo}

Abstract. The present research work was carried out before the COVID-19 pandemic and its objective was to assess the possibilities of application and integration of physical computing, through the programming of sensors and actuators to contribute to the strengthening of knowledge, skills and use of new tools. technologies for teachers of the Electronic Engineering career of the National Technology of Mexico Minatitlán campus, applying the constructionist theory and the Arduino Uno technological tool, practices were designed, and the proof of concepts was applied through a face-to-face workshop. Applying at the end two surveys. From this research, it is concluded that Physical Computing with Arduino Uno under the constructionist approach, strengthens various skills and knowledge related to programming, but also develops tangible skills such as designing, connecting, experimenting and building, as well as skills are favored such as collaboration, computational thinking, creativity, communication, and problem solving.

Keywords: Physical computing, Constructionism, Edmodo

\section{INTRODUCCIÓN}

Seymour Papert creador de la Teoría del aprendizaje Construccionista postuló que el aprendizaje ocurre mejor cuando las personas se involucran en la construcción de un objeto o producto que les apasione, donde el conocimiento se va construyendo de forma activa, a través de la experimentación, simulación, creación y programación de objetos que se puedan compartir. Papert indica que el aprendizaje sucede "felizmente en un contexto donde el alumno está conscientemente comprometido en la construcción de una entidad pública, ya sea un castillo de arena, un programa, un robot o una teoría del universo.” (Papert, 1980).

Por su parte, el término Computación física fue mencionado por primera vez por Dan O’Sullivan y Tom Igoe en el 2004 (citado Przybylla \& Romeike, 2014, p.352) "quienes lo vieron como un elemento crucial de los sistemas que hacen uso de transductores (sensores y actuadores) para conectar lo virtual y el mundo físico. Los productos típicos de la computación fisica son medios tangibles programados". (Przybylla \& Romeike, 2014).

Los autores Dan O'Sullivan y Tom Igoe en su libro (2004) afirman que la computación física permite establecer una conexión entre el mundo físico y el mundo de las computadoras. Siendo el microcontrolador el componente principal que permite interactuar con el mundo físico a través de circuitos de entrada llamados sensores y circuitos de salida llamados actuadores. (Igoe, s.f.).

Actualmente existen varias placas de desarrollo que incluyen un microcontrolador, entra ellas se encuentran: el MakeyMakey, PicoBoard, el Arduino, la Microbit. Para este trabajo de investigación se utilizó la placa de Arduino Uno como componente tecnológico, por su bajo costo, por el número de pines de entradas y salidas que presenta, y el lenguaje de programación muy similar a $\mathrm{C} / \mathrm{C}++$ muy usado en la Ingeniería. 


\section{CONTEXTO}

La investigación se llevó a cabo con docentes de la carrera de Ingeniería Electrónica, implementando la prueba de conceptos mediante un curso denominado "Taller de Cómputo Físico con Arduino Uno", siendo avalado por la academia de la carrera de Ingeniería Electrónica y por el TecNM campus Minatitlán, realizándose los días del 07 al 11 de enero de 2019 en el marco del Programa de Formación y Actualización Docente 2019. El curso se realizó en las instalaciones de la Academia de Ingeniería Electrónica del TecNM campus Minatitlán, donde se cuenta con un espacio con mesas amplias para trabajar.

Al curso asistieron un total de 13 docentes de la Carrera de Ingeniería Electrónica, cuyos perfiles son: 2 profesores con la maestría en Ingeniería Electrónica, 1 profesora y 1 profesor con la Maestría en Ciencias en Ingeniería Electrónica, 1 profesora con la Maestría en Ingeniería Eléctrica con especialidad en bioelectrónica, 1 Doctora en Educación Relacional y bioaprendizaje (con el perfil de Ingeniero Electrónico), 1 profesor es Ingeniero en Comunicaciones y Electrónica, 3 profesores tienen el perfil de Ingenieros Electrónicos y 1 profesor tiene la Maestría en Educación y 2 profesores con el perfil de Ingenieros Electrónicos imparten cátedra en la carrera de Ingeniería en Sistemas Computacionales.

\section{Objetivo del estudio.}

Valorar las posibilidades de aplicación y de integración de la computación física con Arduino Uno, mediante la programación de sensores y actuadores y contribuir con el fortalecimiento de conocimientos, competencias y uso de nuevas herramientas tecnológicas para los docentes de la carrera de Ingeniería Electrónica del TecNM campus Minatitlán y de esta forma contribuir con una educación superior de calidad. (Jiménez, 2020).

\section{Población y muestra.}

En la tabla 1 podemos apreciar la población de docentes de la carrera de Ingeniería Electrónica y la muestra que participó en la investigación durante el curso taller de Cómputo Físico con Arduino Uno.

Tabla 1. Población y muestra con docentes.

\begin{tabular}{|c|c|c|c|c|c|}
\hline Sujeto & $\begin{array}{l}\text { Descripció } \\
\text { n }\end{array}$ & $\begin{array}{l}\text { Poblaci } \\
\text { ón }\end{array}$ & Muestra & Técnica & Instrumento \\
\hline Docentes & $\begin{array}{l}\text { Docentes } \\
\text { de la } \\
\text { Carrera de } \\
\text { Ingeniería } \\
\text { Electrónic } \\
\text { a del } \\
\text { TecNM } \\
\text { campus } \\
\text { Minatitlán }\end{array}$ & 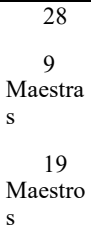 & $\begin{array}{c}13 \\
9 \\
\text { Maestros } \\
4 \\
\text { Maestras }\end{array}$ & Encuesta & $\begin{array}{l}\text { Cuestionario } \\
\text { Lista de Cotejo } \\
\text { de las prácticas } \\
\text { y del proyecto }\end{array}$ \\
\hline
\end{tabular}

\section{DESCRIPCIÓN}

A continuación se describen las actividades realizadas en el presente trabajo de investigación, y los recursos utilizados: metodologías, técnicas y tecnología.

Metodología propuesta.

Para este trabajo de investigación se aplicó una metodología mixta.
Técnica.

Se aplicaron dos encuestas una del TecNM campus Minatitlán con una escala de Likert y una encuesta con preguntas abiertas, que es la que se comparte en la sección de resultados.

\section{Aprendizaje Construccionista.}

Considerando el enfoque de aprendizaje construccionista de Seymour Papert, orientados al aprender haciendo, se diseñaron una serie de prácticas para trabajar la computación física seleccionando la tarjeta Arduino Uno como componente principal; las prácticas se realizaron de forma presencial, consistieron en: construir circuitos, programar, simular y experimentar con diversos componentes electrónicos entre los que se encuentran algunos sensores y actuadores.

Las prácticas diseñadas bajo el enfoque construccionista se implementaron con estudiantes y con docentes, en este artículo se presentan los resultados de la prueba de conceptos realizada con los docentes del TecNM campus Minatitlán, implementadas en el curso: "Taller de Cómputo Físico con Arduino Uno"; cuyo objetivo era medir la percepción de la propuesta y valorar las posibilidades de aplicación y de integración de la computación física con Arduino Uno en las asignaturas, y contribuir con el fortalecimiento de conocimientos, competencias y uso de nuevas herramientas tecnológicas para los docentes. (Jiménez, 2020).

El curso tuvo con una duración de $30 \mathrm{~h}$, impartidas en cinco sesiones.

\section{Tecnología propuesta.}

Como componente tecnológico se trabajó con la computación física con Arduino Uno para los fundamentos de la programación, bajo el enfoque construccionista. Arduino es una plataforma electrónica de código abierto basada en hardware y software utiliza el lenguaje de programación Arduino y el software Arduino IDE (Arduino, 2018). Esta placa de desarrollo contiene un microcontrolador como componente principal encargado de procesar las instrucciones guardadas en su memoria. Los Microcontroladores "son los que permiten interactuar con el mundo físico que nos rodea a través de circuitos de entrada llamados sensores y circuitos de salida llamados actuadores, generalmente se reserva para un procesador simple que hace una sola tarea, como escuchar sensores" (Igoe, 2014). Estos elementos son programados a través de una computadora, haciendo de la programación abstracta una forma tangible de programar.

En el curso taller las prácticas se implementaron de forma física, pero fueron diseñas usando el entorno de Tinkercad, para experimentar y programar también de forma virtual.

También se trabajó con la plataforma de Edmodo que es una plataforma educativa que administra un aula virtual, aloja y comparte materiales de clase y permite que el aprendizaje sea accesible en cualquier lugar (Edmodo, s.f.). Permitiendo alojar y compartir los materiales del curso (diapositivas, videos, enlaces), dar las instrucciones de las prácticas a realizar y recopilar las evidencias realizadas por los docentes participantes; alojando al final la encuesta que se aplicó a los docentes. Siendo esta plataforma una forma extra de comunicación y colaboración con los docentes y entre los docentes participantes más allá del aula. 
Se les proporcionó a los profesores el enlace a la plataforma de Edmodo, los profesores se dieron de alta proporcionando un correo electrónico, y una contraseña; los docentes se unieron al curso virtual por medio de una clave proporcionada en la primera sesión.

En la Figura 1, podemos apreciar una pantalla del curso diseñado en la plataforma Edmodo, en ella se muestran las carpetas donde se alojaron los materiales y presentaciones del curso, estas se distribuyeron en sesiones, una por cada día.

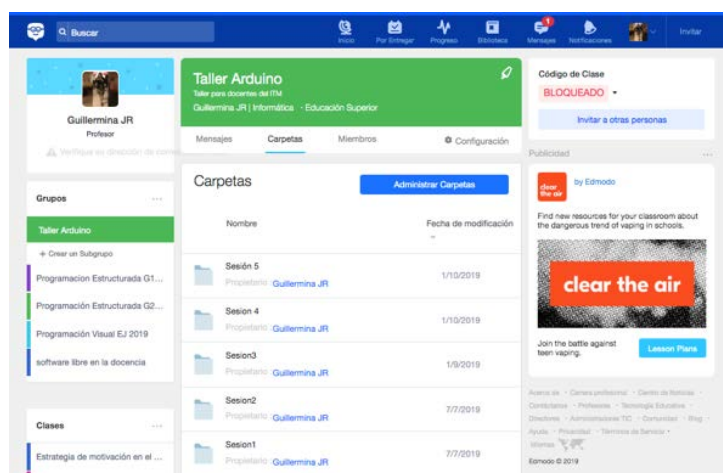

Figura 1. Curso alojado en la Plataforma de Edmodo.

\section{Prueba de conceptos con docentes}

La prueba de conceptos de la computación física con Arduino bajo el enfoque construccionista se llevó a cabo con 13 docentes del Instituto Tecnológico de Minatitlán, durante el curso "Taller de Cómputo Físico con Arduino Uno".

La primera sesión del taller, consistió en dar a conocer a los profesores el paradigma construccionista y el paradigma de la computación física, se describieron los componentes de la tarjeta del Arduino Uno, y se compartió un video de la página principal del Arduino. Se dieron a conocer los conceptos de sensores y actuadores mostrando algunos ejemplos.

Se utilizó la plataforma de Edmodo para compartir los materiales.

Se solicitó a los docentes seleccionar un sensor y un actuador de los mostrados en la sesión y se solicitó investigarán sus características. Presentando en equipos los resultados encontrados.

Durante las siguientes sesiones se realizaron con una serie de prácticas de la computación física con Arduino Uno, donde los docentes pudieron conectar, programar y experimentar con diversos objetos para pensar desde la tarjeta de Arduino y algunos sensores y actuadores. Implementando las siguientes prácticas:

1. Hola mundo - prender y apagar un led

2. Programación de una secuencia de encendido de ocho leds.

3. Programación de entradas digitales con un pulsador para encender leds

4. Programación de un semáforo digital

5. Programación de una carita feliz

6. Programación de un sensor (potenciómetro) para variar el brillo de un led
7. Programación de un motor de CD (actuador)

8. Programación de un servomotor SG90 (actuador)

Todas estas prácticas permitieron a los docentes construir circuitos y programar sensores y actuadores, con lo cual podían relacionar los conceptos abstractos de la programación con el actuar de objetos reales y tangibles.

En la Figura 2 podemos apreciar a los docentes conectando, programando y trabajando de forma colaborativa.

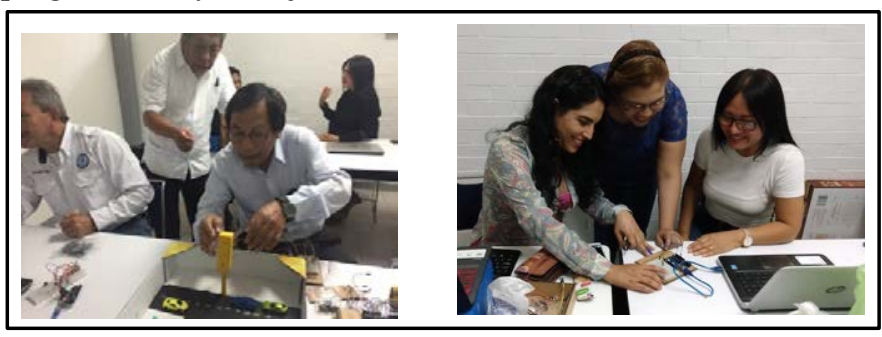

Figura 2. Docentes realizando prácticas de la computación física con Arduino Uno.

En la Figura 3, podemos apreciar un colash fotográfico de las prácticas realizadas por los docentes usando la computación física con Arduino Uno.
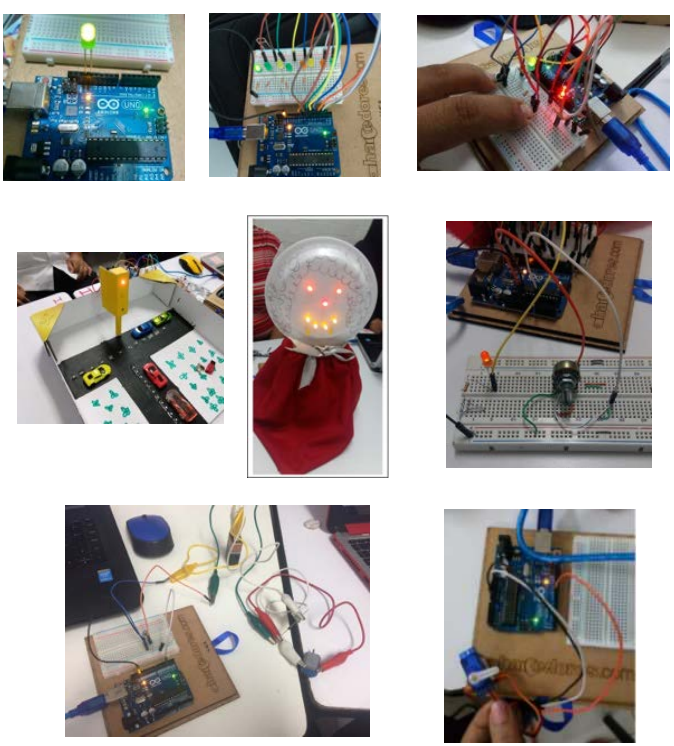

Figura 3. Prácticas realizadas por los docentes.

Trabajo colaborativo y proyectos realizados.

Siguiendo el enfoque construccionista de Seymour Papert, los docentes se involucraron en el desarrollo de un proyecto final, escogido por ellos y que les apasionara; trabajaron en equipos, aplicaron lo visto en las sesiones y resolvieron problemas en conjunto. Presentando en la última sesión sus creaciones. A continuación se muestran algunos de los proyectos creados, que representan sistemas didácticos interactivos mediante la computación física con Arduino Uno.

En la Figura 4 podemos apreciar el proyecto "sistema didáctico con el Arduino Uno para controlar el Nivel de un tanque", mediante un sensor ultrasónico, usando también diversos componentes electrónicos existentes en el mercado. 


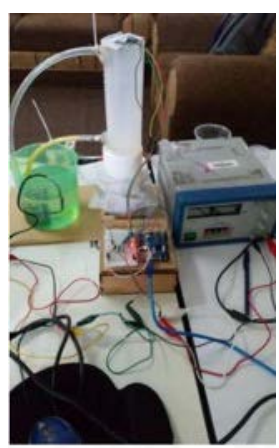

Figura 4. Proyecto para controlar el nivel de un tanque.

Este proyecto se presentó en el Congreso internacional de investigación Academia Journals Oaxaca 2019 con el tema "Sistema de control de nivel On-Off con Arduino Uno", cumpliendo de esta forma con otra competencia docente que es la divulgación científica.

Otro de los proyectos realizados fue la creación de un juego didáctico interactivo y lúdico, usando la computación física con Arduino Uno, denominado "Ruleta de asignación de actividades". El juego consistía en que al ser presionado un push button, por el estudiante, girara un motor previamente programado con el Arduino Uno, el cual a su vez hace girar la ruleta de actividades que se encuentra unido a éste; al soltar el estudiante el botón pulsador, el motor comienza a detenerse poco a poco ocasionando que la ruleta se detenga al azar en una actividad determinada por el docente, asignándose al estudiante dicha actividad. En la Figura 5 podemos apreciar la ruleta de actividades creada y programada por las docentes.

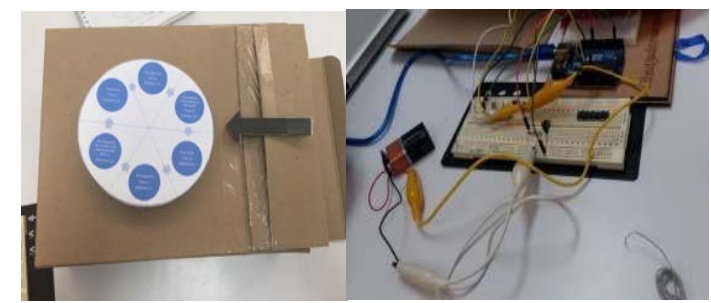

Figura 5. Proyecto ruleta de asignación de actividades.

El tercer proyecto presentado por los docentes fue un sistema de control de llenado de agua para un tinaco (simulación) considerando el nivel de agua en la cisterna, ver Figura 6; haciendo una simulación de encendido leds que marcaban el nivel bajo y el nivel alto del tinaco y de la cisterna, así como también la simulación del encendido y apagado de una bomba.

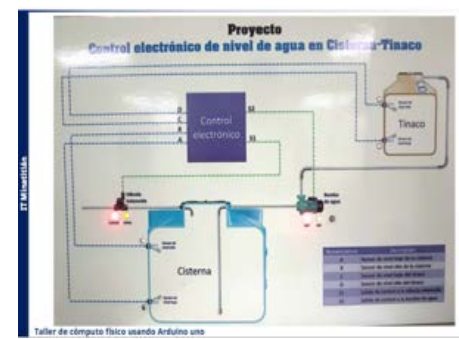

Figura 6. Proyecto Simulación del control del llenado de un tinaco.
El último proyecto presentado consistió en medir la temperatura ambiente y mostrarla en grados centígrados en la pantalla de la computadora, como se aprecia en la figura 7.

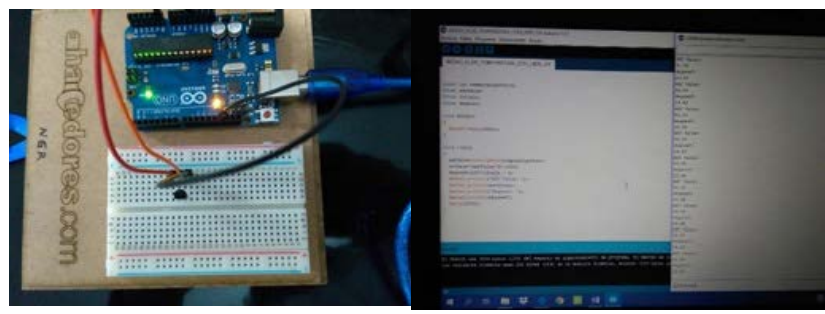

Figura 7. Proyecto Medidor de temperatura ambiental.

\section{Resultados}

Impacto en docentes de la computación física bajo el enfoque construccionista.

Los docentes que realizaron el proyecto para controlar el nivel de un tanque expresaron: "Gracias a la implementación de la interfaz realizada con Arduino uno se logró adaptar un juego de tanques a un sistema didáctico que servirá para realizar diferentes prácticas de laboratorio de control automático para fomentar la innovación y el crecimiento tecnológico en la Academia, permitiendo realizar diversas pruebas donde los estudiantes la pueden poner en práctica fácilmente determinando lo que están haciendo, entendiendo el concepto de control On-Off y la gran importancia que tiene los sistemas de medición y control en la industria."

El equipo de que presentó el proyecto "Sistema de control de llenado de agua para un tinaco expresó que "la etapa de control electrónico se implementó usando la computación física con Arduino Uno, reemplazó una implementación anterior con circuitos integrados de MSI (Mediana Escala de Integración), resultando en un costo mucho menor y menos componentes electrónicos".

Por su parte el equipo de maestras que presentó el proyecto de la "Ruleta de asignación de actividades", expresó que la actividad las puso a pensar, y en buscar una forma dar solución a su propuesta, pudiendo finalmente llevarla a cabo relacionando la práctica de los actuadores y su forma de programarlo.

En la Figura 8 podemos apreciar la relación de la Computación física y el construccionismo con las competencias docentes que se favorecieron durante el curso, fortaleciendo algunas competencias tecnológicas como la programar, simular, usar software para crear, compartir y comunicar; se favorecieron las competencias duras o tangibles como la de conectar, construir y diseñar y las competencias suaves como la resolución de problemas, el trabajo en equipo, la comunicación, creatividad, la investigación y la divulgación científica.

En relación a las competencias computacionales se favorecieron algunos de los elementos del pensamiento computacional (ver Valenzuela, 2020) tal como la descomposición de un problema en partes pequeñas y manejables, la abstracción al crear un modelo mental, crearlo en físico, y la resolución de problemas mediante el diseño de algoritmos e instrucciones; en las prácticas y proyectos se programaron variables y las estructuras de control secuencial, selectivas y las repetitivas o iterativas principalmente. Se dieron a conocer algunas instrucciones para la programación de sensores tal como el sensor de temperatura y el sensor 
ultrasónico; y las instrucciones para la programación de actuadores como leds, motores y servomotores.

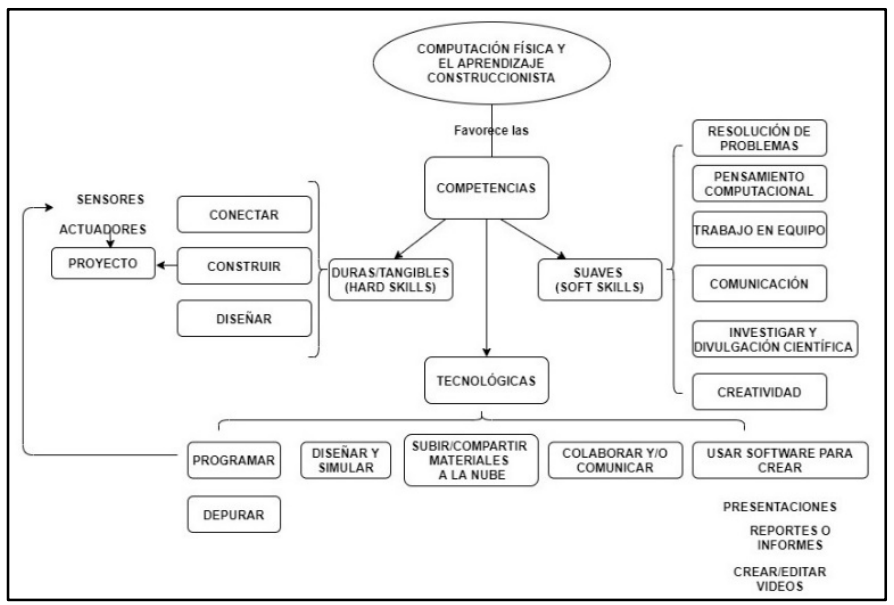

Figura 8. La computación fisica y el construccionismo y su relación con las competencias docentes. (Diseño propio).

Evaluación del impacto de la computación física bajo el enfoque construccionista.

Se aplicaron dos encuestas para evaluar el impacto, una con escala de Likert (Tecnológico) y otra encuesta con preguntas abiertas que se comparte a continuación. La encuesta aplicada (Jiménez, G., 2020), se subió al curso creado en la plataforma del Edmodo, donde los docentes pudieron descargarlo, contestarlo y enviar la respuesta en la misma plataforma.

Resultados de la encuesta aplicada a los profesores

A continuación se muestra el concentrado de las preguntas y las respuestas proporcionadas por los docentes a la encuesta aplicada:

1. ¿Considera que el cómputo físico aporta o favorece el desarrollo del pensamiento computacional en los estudiantes de la carrera de Ingeniería Electrónica?

- Los trece docentes participantes afirmaron que el Cómputo físico con Arduino Uno, si favorece el pensamiento computacional de los estudiantes de la carrera de Ingeniería Electrónica

- Un docente comenta que se debe acompañar de la investigación documental de cómo operan o funcionan los componentes, para que no sea mecanizando.

- Otro docente afirma permite adquirir habilidades constructivas

- $\quad$ Otro docente nos comparte que aplicar el Cómputo físico con Arduino Uno coadyuva a fomentar la lógica en la programación aplicada a un sistema que utiliza los sensores y actuadores.

2. ¿Considera que las prácticas con el cómputo físico con Arduino Uno favorece el perfil de un Ingeniero Electrónico? Si, No y Porqué

- Los trece docentes participantes afirman que las prácticas con el Cómputo físico con Arduino Uno si favorece al perfil de un Ingeniero electrónico.

- Además, que contribuye al logro de las competencias que marca el perfil del ingeniero
Electrónico del TecNM cubriendo las competencias:

“Aplica las nuevas Tecnologías de la Información y la Comunicación, para la adquisición y procesamiento de datos". "Desarrollar y administrar proyectos de investigación y/o desarrollo tecnológico." "Utilizar lenguaje de descripción de hardware y programación de microcontroladores en el diseño de sistemas digitales para su aplicación en la resolución de problemas".

- El cómputo físico con Arduino Uno permite relacionar lo aprendido electrónicamente con las aplicaciones.

- Pueden ver los resultados de la programación en físico

- Puede contribuir con soluciones del entorno

- Las habilidades adquiridas con el Cómputo físico y las aplicaciones otorgadas con él, pueden emplearlo en cualquiera de los experimentos de las diferentes materias o asignaturas.

- Las prácticas mediadas con el cómputo físico hacen que las materias sean más prácticas y demostrativas

3. ¿Qué otras prácticas del cómputo físico con Arduino Uno le gustaría que se implementaran en el curso?

La mayoría de los docentes se mostró interesado y expresaron que les gustaría en próximos cursos que se incluyeran más temas relacionados con sensores y actuadores como los servomotores y motores de paso, 1 docente expresó que le gustaría realizar una práctica mediante comunicación inalámbrica, otro docente expresó hacer prácticas usando displays.

4. Algo más que le gustaría expresar en relación al cursoTaller de Cómputo físico con Arduino uno.

- 5 docentes comentaron que resultó “excelente” y uno pidió continuación y otro más manifestó que el curso despertó en el "el gusanito de realizar proyectos con Arduino Uno",

- 3 docentes comentaron que estuvo "muy interesante", además que es una puerta abierta a la imaginación, y manifestaron sentirse "incentivados y/o "motivados" a seguir adquiriendo más conocimientos sobre este importante tema y de esta manera mejorar su práctica docente.

- 1 docente mencionó que le gustaría que se manejara como un taller, tal vez informal, es decir, de manera voluntaria, como un club en donde se pudiera expresar dudas y aportaciones, que permita el crecimiento de esta aplicación.

- 1 docente manifestó haberse divertido mucho en el curso-taller

- 1 docente afirmo que es muy importante para el desarrollo de las materias de electrónica.

- 1 docente expresó que estuvo muy bueno el curso, así como no los impartió la instructora. 
- 1 docente expreso ¡Gracias por su entusiasmo!

\section{CONCLUSIONES}

De esta investigación, se concluye que la Computación física con Arduino Uno fortalece diversas competencias y conocimientos relacionados a los fundamentos de la programación, pero también se desarrollan habilidades o competencias tangibles como las de diseñar, conectar y construir, así como diversas habilidades o competencias suaves tal como la resolución de problemas, el pensamiento computacional, el trabajo en equipo, la creatividad, la comunicación e investigación y divulgación de las ciencias.

De los datos recopilados de los docentes, se obtiene una valoración positiva a la aplicación e integración de la computación física bajo el enfoque del construccionismo, el cual contribuyó con el fortalecimiento de conocimientos, competencias y uso de nuevas herramientas tecnológicas para los docentes de la carrera de Ingeniería Electrónica.

Durante el curso taller de Cómputo Físico con Arduino Uno se obtuvieron comentarios positivos, entre los cuales mencionaron les resultó excelente, e interesante; es una puerta abierta a la imaginación, los docentes diseñaron, construyeron tecnología educativa tangible para sus estudiantes. Manifestaron sentirse "incentivados y "motivados" a seguir adquiriendo más conocimientos sobre este importante tema y de esta manera mejorar su práctica docente.

Los temas de la computación física pueden ser transferibles a diversos niveles educativos, pudiendo variar los productos desarrollados, ya que estos parten de la creatividad de cada uno de los participantes e instructores y de lo que desean crear.

Con este curso taller los profesores pudieron usar herramientas tecnológicas para programar, pero también utilizaron la plataforma tecnológica de Edmodo para alojar y compartir materiales de forma más fácil, trabajar de forma colaborativa y tener una comunicación adicional; funcionando también como un repositorio de evidencias; dando una experiencia de tipo B-Learning al combinar el entorno presencial y el virtual. (Jiménez, G., 2020)

Para algunos profesores fue su primera experiencia en el uso de la plataforma tecnológica del Edmodo, y comentaron que les gustaría conocer más sobre la plataforma. A continuación se presentan algunos comentarios de los profesores sobre el uso del Edmodo.

"El Edmodo fue una herramienta atractiva para la entrega de reportes, imágenes y videos a través de publicación en mensajes o a través de respuestas a la tareas publicadas. Permitió que las entregas estuvieran organizadas y con fecha límite, lo que ayuda al docente a administrar las recepciones de dichas entregas".

"En el curso de Taller de cómputo físico con Arduino Uno, la instructora MTE. Guillermina Jiménez Rasgado, nos proporcionó la información a través de la plataforma de Edmodo de las prácticas a desarrollar, esta plataforma es muy sencilla y me pareció muy similar al Facebook, lo importante es que la instructora del curso llevó la batuta del aula virtual. Además cada grupo de trabajo incluyó en el muro la información de nuestras prácticas para compartir nuestra información, comentarios y experiencia al realizarlas”.
"Fue realmente muy práctico y amigable la interacción con el Edmodo".

"El Edmodo es una plataforma muy útil, tiene muchas herramientas para el proceso educativo, no he aprendido a usarlas todas, pero fue una experiencia agradable en el curso de Arduino".

"El Edmodo es bueno, ya que sin ver al instructor, sientes el compromiso de no fallar, en las tareas pendientes por cumplir, $y$ te permite, intercambiar información con tus otros compañeros".

"Edmodo me pareció práctico porque ya lo había usado en otros cursos".

Para el taller de computación físicas, se recomienda conseguir con anticipación los materiales y en un mejor momento equipar los laboratorios con elementos de la computación física, para que docentes y estudiantes puedan construir, experimentar, programar. Conforme más prácticas se realicen, se podrán agregar más componentes relacionados a la computación física y la programación de sensores y actuadores y contribuir en la resolución de problemas del entorno y sociedad.

\section{AgRAdeCIMIENTOS}

Agradecemos al Tecnológico Nacional de México - campus Instituto Tecnológico de Minatitlán directivos y docentes participantes, por el apoyo brindado para llevar a cabo este trabajo de investigación.

Agradecemos el apoyo del Instituto Politécnico Nacional y de la Red LaTE Mx.

\section{REFERENCIAS}

Arduino (2018). Página Oficial. Recuperado de https://www.arduino.cc/en/Guide/Introduction

Edmodo (s.f.). Página oficial. Recuperado de https://new.edmodo.com/?language $=$ es

Jiménez, Guillermina (2020). Didáctica de la programación basada en computación física y el construccionismo, para favorecer el pensamiento computacional en Ingeniería Electrónica del TECNM CAMPUS MINATITLÁN. (Tesis Doctoral). UNIVDEP, México.

Igoe (s.f.). Computación física ITP. Microcontroladores: conceptos básicos. Recuperado de https://itp.nyu.edu/physcomp/lessons/microcontrollersthe-basics/

O'Sullivan, D., \& Igoe, T. (2004). Physical Computing: Sensing and Controlling the Physical World with Computers. Thomson Course Technology PTR, Boston.

Przybylla, M., \& Romeike, R. (2014). Key Competences with Physical Computing. In Proceedings of Key Competencies in Informatics and ICT 2014, pp. 351-361

Papert, S. (1980). Mindstorms. Nueva York: Basic Books.

Valenzuela, J. (22 de Septiembre de 2020). ISTE. Como desarrollar pensadores computacionales. Recuperado de https://www.iste.org/es/explore/how-developcomputational-thinkers 